\title{
dc-conduction mechanism and Peierls gap in organic and inorganic charge-density-wave conductors
}

\author{
W. Brütting, P. H. Nguyen, and W. Rieß \\ Physikalisches Institut und Bayreuther Institut für Makromolekülforschung, Universität Bayreuth, \\ 95440 Bayreuth, Germany \\ G. Paasch \\ Institut für Festkörper- und Werkstofforschung, 01171 Dresden, Germany
}

(Received 21 November 1994)

\begin{abstract}
It has been shown in a recent analysis of the temperature dependence of the dc conductivity of the quasi-one-dimensional conductor (fluoranthene) ${ }_{2} \mathrm{PF}_{6}$ that in spite of the occurrence of the Peierls transition to a charge-density-wave ground state (formally implicating polarons as excitations), the dc conduction is essentially due to electron-hole transport in bands and acoustical phonon scattering of the carriers. The theory allows for the determination of the temperature dependence of the Peierls gap below and the fluctuating pseudogap above the transition temperature. Our dc-conductivity measurements confirm that a common temperature dependence occurs in organic radical cation salts and in inorganic materials from the groups of the blue bronzes and the transition metal tetrachalcohalogenides. These materials are rather different especially with respect to the nature of the states forming the conduction band and the filling of the latter. Here we reduce the needed information on the band structure to a minimum connected with optical data and extend the theory to the case of a gap small compared to $k_{B} T$. The theory is applied to $(\mathrm{Fa})_{2} \mathrm{PF}_{6}, \mathrm{~K}_{0.30} \mathrm{MoO}_{3}$, and $\left(\mathrm{TaSe}_{4}\right)_{2} \mathrm{I}$ as representatives of the above-mentioned groups of materials. From the measured conductivity data the temperature dependence of the Peierls gap below and the pseudogap above the transition temperature are determined as well as several conductivity-related quantities. Similarities and differences of the investigated materials are discussed.
\end{abstract}

\section{INTRODUCTION}

A variety of quasi-one-dimensional (Q1D) systems show instabilities due to electron-phonon and electronelectron interactions leading to numerous interesting properties such as bond-order waves, charge-density waves (CDW's), ${ }^{1,2}$ and spin-density waves. ${ }^{3,4}$ The Peierls instability against lattice distortions leads to the opening of the Peierls gap below the transition temperature and to a CDW ground state in different inorganic systems such as linear-chain platinum compounds, ${ }^{5}$ several transition-metal compounds, ${ }^{5}$ the blue molybdenum bronzes, ${ }^{6}$ and also organic radical-cation salts such as (fluoranthene) ${ }_{2} X\left(X=\mathrm{PF}_{6}, \mathrm{AsF}_{6}, \mathrm{SbF}_{6}\right),{ }^{7}$ (perylene $)_{2} X^{8}{ }^{8}$ and (perylene $)_{2} M(\mathrm{mnt})_{2} \quad(M=\mathrm{Ni}, \mathrm{Cu}$, $\mathrm{Pd}, \mathrm{Pt}, \mathrm{Au}, \mathrm{Fe}, \mathrm{Co} ; \mathrm{mnt}=$ maleonitriledithiolate $).{ }^{9}$

A central feature of the Peierls transition is the appearance of fluctuation effects. ${ }^{10}$ In a strictly $1 \mathrm{D}$ system fluctuations of the order parameter would prevent a phase transition at a finite temperature. However, in a real Q1D system residual three-dimensional interactions partially suppress the effect of such fluctuations, so that a Peierls transition can take place at a finite temperature $T_{P}$. The temperature dependence of the Peierls gap below $T_{P}$ is usually described as a scaled mean-field dependence for a system with a half-filled undisturbed conduction band ${ }^{11}$ (the dependence on the band filling is considered in Ref. 12). Above the transition temperature $T_{P}$ there remains a fluctuating pseudogap which finally vanishes at some higher temperature of the order of the mean-field transition temperature $T_{P}^{M F}$; however, the theoretically determined density of states of the fluctuating gap ${ }^{10}$ has not been seen directly in experiments. On the other hand, various experiments such as $\mathrm{x}$-ray and neutron scattering, ${ }^{13,14}$ magnetic susceptibility measurements, ${ }^{15,16}$ and optical investigations ${ }^{17,18}$ have given evidence for the presence of CDW fluctuations in Q1D conductors. Thus the independent determination of the temperature dependence of the gap below and above the transition temperature directly from dc-conductivity data can provide additional information about fluctuation effects in Q1D CDW conductors.

The occurrence of the Peierls gap implies that the excitations of the system are polaronic coupled electronlattice excitations. On the other hand, we have shown in a recent analysis of the dc conductivity of $(\mathrm{Fa})_{2} \mathrm{PF}_{6}$ that the transport is adequately described by simple electronic band-to-band excitations. ${ }^{19}$ This implies that the combination of three-dimensional effects and fluctuations is still of such a magnitude that the Peierls state occurs, but that the excitations determining transport can be described effectively as single-particle excitations from the valence to the conduction band with acoustical deformation potential scattering as the dominating scattering process. It has also been shown that one can determine 
the temperature dependence of the gap with this description by using experimental dc-conductivity data.

Published conductivity data indicate a similar temperature dependence of the dc conductivity in fluoranthene radical cation salts ${ }^{20}$ the blue molybdenum bronzes, ${ }^{21}$ and some transition-metal tetrachalcohalogenides. ${ }^{22}$ These groups of substances are rather different since wave functions of different states form the Q1D system leading to the Peierls instability and also the band filling in the metallic state above the transition is different. The aim of the present paper is at first to confirm this similarity of the dc conductivity by our measurements and second to compare these substances by applying basically our theoretical description. This requires several modifications of the theory. In Ref. 19 we used a special band structure model for a half-filled conduction band in the metallic state. The formulation given here reduces the needed information on the band structure and is independent of the band filling by relating the band structure parameter to optical data. Further, contrary to the previous formulation, the nonparabolicity of the bands is considered, which becomes important if the gap is less than $k_{B} T$. Also the possibility is taken into account that scattering at higher energetic nonpolar optical phonons must be considered. Finally, to obtain from the measured conductivity not only the temperature-dependent gap but also related quantities such as the mean relaxation time, the mean free path, the mobility, and the velocity of sound, appropriate formulations must be introduced considering both the nonparabolicity of the bands and an arbitrary degree of degeneration.

\section{MATERIALS AND THEIR dc CONDUCTIVITY}

The materials investigated here are linear-chain compounds, where the basic structural units are organic or inorganic groups forming chains with strongly overlapping electronic wave functions along the chains and weak overlap in the perpendicular direction. This leads to a Q1D electronic band structure with relatively wide bands in the chain direction and only little dispersion perpendicular to it. Consequently, the resulting Fermi surface shows nesting properties, which are the prerequisite for the Peierls transition to a CDW ground state.

In the following, the structural and electronic properties of the three systems will be briefly discussed; for details we refer to Ref. 19 in the case of $(\mathrm{Fa})_{2} \mathrm{PF}_{6}$ and Refs. 23 and 6 for $\left(\mathrm{TaSe}_{4}\right)_{2} \mathrm{I}$ and $\mathrm{K}_{0.30} \mathrm{MoO}_{3}$, respectively. $(\mathrm{Fa})_{2} \mathrm{PF}_{6}$ belongs to the class of arene radical cation salts $(\mathrm{Ar})_{2} X$, where $\mathrm{Ar}$ is an aromatic hydrocarbon such as fluoranthene $\left(\mathrm{C}_{16} \mathrm{H}_{10}\right)$ or perylene $\left(\mathrm{C}_{20} \mathrm{H}_{12}\right)$ and $X$ is a monovalent anion $\left(X^{-}=\mathrm{PF}_{6}^{-}, \mathrm{AsF}_{6}^{-}, \mathrm{SbF}_{6}{ }^{-}\right)$. The orbitals forming the bands are the $\pi$ orbitals of the aromatic molecule, but it must be mentioned that the $1 \mathrm{D}$ chain is formed by a $\sigma$-type binding perpendicular to the molecular in-plane $\pi$-electron system. Due to a charge transfer of 0.5 electrons per Fa molecule and a slight dimerization within the Fa stack, the unit cell contains two Fa molecules and the conduction band is half filled.

$\left(\mathrm{TaSe}_{4}\right)_{2} \mathrm{I}$ is a member of the halogenated transition- metal chalcogens $\left(M X_{n}\right)_{m} Y$, where $M$ stands for the transition metal $\mathrm{Ta}$ or $\mathrm{Nb}, X$ for the chalcogen $\mathrm{S}$ or Se, and $Y$ can be the halogen I, Br, or Cl. Here the bands are formed from the Ta $d$ orbitals in the $\mathrm{TaSe}_{4}$ chains, which are separated by $\mathrm{I}^{-}$ions. The charge transfer is again 0.5 electrons per $\mathrm{Ta}$, but because of a tetramerization of the chain the band filling is $1 / 4$.

Another group of linear-chain compounds with a CDW ground state, of which $\mathrm{K}_{0.30} \mathrm{MoO}_{3}$ is the prime example, are the blue molybdenum bronzes $A_{0.30} \mathrm{MoO}_{3}$ with $A=$ $\mathrm{K}, \mathrm{Rb}$, or $\mathrm{Tl}$. The structure can be viewed as infinite chains of $\mathrm{MoO}_{6}$ octahedra sharing corners along the chain direction. Band structure calculations ${ }^{24}$ show that the conduction band consists of two almost parallel bands with each of them three-quarters filled.

Meanwhile the Peierls transition to a CDW ground state has been detected via $\mathrm{x}$-ray investigations in all three compounds by the appearance of satellite reflections below $T_{P}$. While in the case of $\left(\mathrm{TaSe}_{4}\right)_{2} \mathrm{I}$ and $\mathrm{K}_{0.30} \mathrm{MoO}_{3}$ the wave vector of the superlattice spots is incommensurate with the reciprocal lattice, ${ }^{13}$ in $(\mathrm{Fa})_{2} \mathrm{PF}_{6}$ it has been shown recently ${ }^{14}$ that $q_{S}=\frac{1}{2} a^{*}$, which means that the CDW is pinned by commensurability with the lattice.

In past years the materials have been characterized by a variety of methods; most important in this context are dc-conductivity and magnetic susceptibility measurements together with polarized optical reflectance spectra, from which band structure parameters are derived. In order to compare the three substances, the temperature dependence of the electrical conductivity of the inorganic CDW conductors $\left(\mathrm{TaSe}_{4}\right)_{2} \mathrm{I}$ and $\mathrm{K}_{0.30} \mathrm{MoO}_{3}$ has been reinvestigated with the same experimental setup as described earlier for $(\mathrm{Fa})_{2} \mathrm{PF}_{6} \cdot{ }^{20}$ The data for $\left(\mathrm{TaSe}_{4}\right)_{2} \mathrm{I}$ and $\mathrm{K}_{0.30} \mathrm{MoO}_{3}$ are in good agreement with the results achieved by other groups ${ }^{21,22}$ and indicate a similarity of the underlying conduction mechanism in all three substances.

Figure 1(a) shows the temperature dependence of the dc conductivity of the three systems between room temperature and about $25 \mathrm{~K}$. All curves show qualitatively the same behavior; however, there are characteristic differences in the Peierls transition temperature $T_{P}$, the room temperature conductivity, and the ground-state energy gap $2 \Delta(0)$ (see Table I). At room temperature the systems show relatively high conductivities of typically $10^{3}(\Omega \mathrm{cm})^{-1}$; however, apart from the blue bronze no increase of conductivity with decreasing temperature is observed, as should be expected for a Q1D metal. At the Peierls transition the slope of $\sigma(T)$ changes abruptly and, for temperatures below $T_{P}$, the conductivity rapidly decreases due to the opening of a BCS-like energy gap at the Fermi level. At even lower temperatures the conductivity follows approximately a thermally activated law with $\sigma(T) \propto \exp \left[-\Delta(0) / k_{B} T\right]$. From the Arrhenius plot in Fig. 1(b) the characteristic energy gap $2 \Delta(0)$ for each system can be determined. At temperatures below about $50 \mathrm{~K}$ deviations from the Arrhenius law are observed due to defects contributing to the conductivity, which will not be considered here.

Remarkable is the temperature dependence of the con- 
ductivity in the range above $T_{P}$. Although there are relatively high conductivities, $(\mathrm{Fa})_{2} \mathrm{PF}_{6}$ and $\left(\mathrm{TaSe}_{4}\right)_{2} \mathrm{I}$ show no metallic behavior. This is due to pretransitional fluctuations of the CDW ground state, which lead to a pseudogap in the electronic density of states. In the case of the blue bronze, however, an increase of the conductivity with decreasing temperature is observed. A detailed analysis (Fig. 2) shows that above approximately $225 \mathrm{~K}$ the resistivity $\rho=1 / \sigma$ is proportional to the temperature, which is the behavior of a conventional metal at higher temperatures. Thus we have to expect a very small gap above $T_{P}$, which should vanish completely above $225 \mathrm{~K}$.

Following the above explanations the overall temperature dependence of $\sigma(T)$ can be described qualitatively with a temperature-dependent energy gap as shown in Fig. 3 and discussed previously in Ref. 19. For $T<$ $T_{P}$ the Peierls gap $2 \Delta_{\mathrm{MF}}(T)$ shows a scaled meanfield behavior; ${ }^{25}$ however, in the BCS relation $2 \Delta(0)=$ $3.5 k_{B} T_{P}^{\mathrm{MF}}$, in the case of the real Peierls transition temperature $T_{P}$, a constant of the order of $4-11$ must be used instead of 3.5. Above $T_{P}$ the pseudogap $\Delta_{\text {eff }}(T)$ is
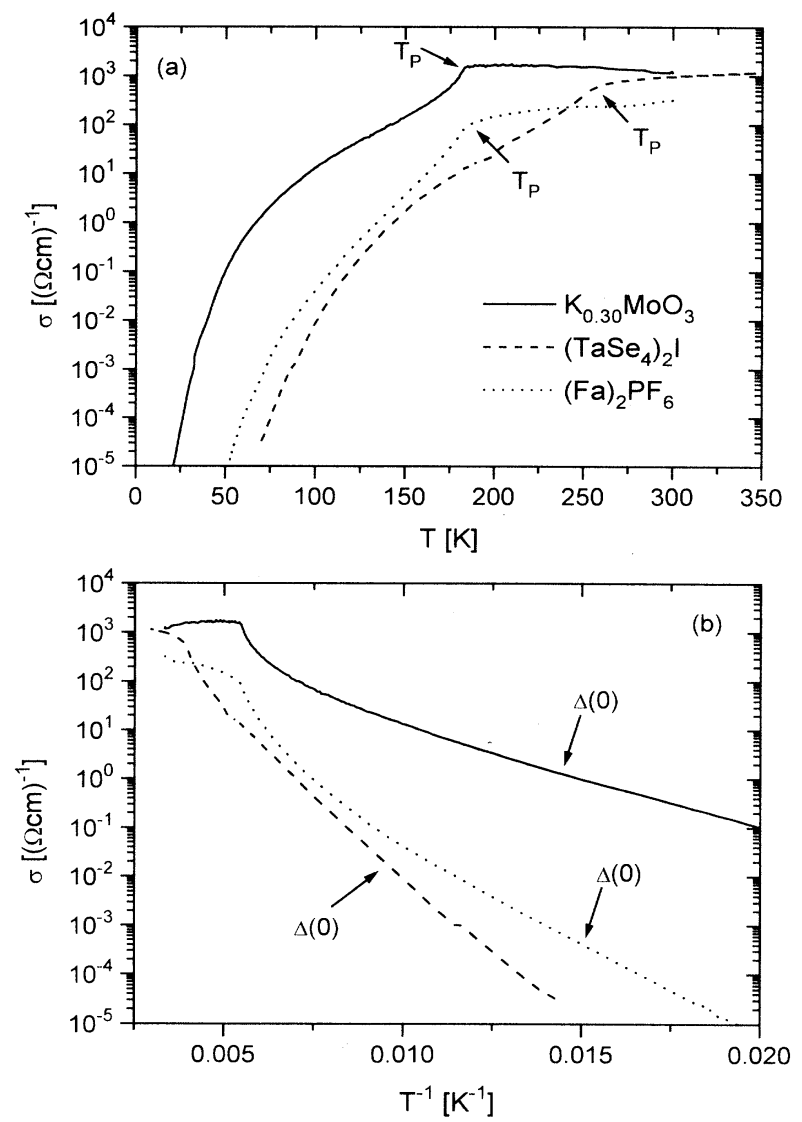

FIG. 1. (a) Temperature dependence of the dc conductivity (measured along the highly conducting 1D crystal axis) for the three CDW systems investigated. The respective Peierls transitions are indicated by arrows. Typical parameters for the systems are given in Table I. (b) In the Arrhenius plot the energy gap $2 \Delta(0)$ in the CDW ground state can be determined from the thermally activated behavior indicated by arrows.
TABLE I. Parameters characterizing the three CDW systems. For the low temperature activation energy $\Delta(0)$ and the room temperature conductivity $\sigma(300 \mathrm{~K})$, the values can vary from crystal to crystal. The typical intervals of the values found in our experiments are given. The mean-field transition temperature is calculated from the BCS relation $2 \Delta(0)=3.5 k_{B} T_{P}^{\mathrm{MF}}$ and varies with the values of $\Delta(0)$.

\begin{tabular}{lccc}
\hline \hline Parameter & $(\mathrm{Fa})_{2} \mathrm{PF}_{6}{ }^{\mathrm{a}}$ & $\left(\mathrm{TaSe}_{4}\right)_{2} \mathrm{I}^{\mathrm{b}}$ & $\mathrm{K}_{0.30} \mathrm{MoO}_{3}{ }^{\mathrm{c}}$ \\
\hline$T_{P}(\mathrm{~K})$ & 182 & 263 & 183 \\
$\Delta(0)(\mathrm{meV})$ & $60-90$ & $130-140$ & $30-40$ \\
$T_{P}^{\mathrm{MF}}(\mathrm{K})$ & $400-600$ & $860-920$ & $200-260$ \\
$\sigma(300 \mathrm{~K})\left[(\Omega \mathrm{cm})^{-1}\right]$ & $10^{2}-10^{3}$ & $10^{3}$ & $10^{2}-10^{3}$ \\
$\sigma_{\|} / \sigma_{\perp}(300 \mathrm{~K})$ & $10^{4}$ & $10^{3}$ & $100: 10: 1$ \\
Band filling & $1 / 2$ & $1 / 4$ & $3 / 4$ \\
\hline \hline
\end{tabular}

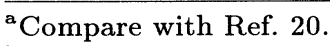

${ }^{\mathrm{b}}$ Compare with Ref. 5 .

${ }^{\mathrm{c}}$ Compare with Ref. 6.

slowly decreasing with increasing temperature. Figure 3 shows the behavior of $\Delta_{\text {eff }}(T)$ as derived from paramagnetic susceptibility by Johnston et al. ${ }^{26}$ However, the gap derived from $\chi_{\text {para }}$, which is an equilibrium quantity, and the transport gap relevant for $\sigma(T)$ can differ considerably.

Therefore, in the following, a theory will be developed that allows a direct determination of the gap from conductivity data. From the application of the theory to the three systems differences and common features will be elaborated. Other related transport quantities also will be discussed.

\section{THEORY}

In this section we generalize the description given in Ref. 19 for the dc transport in $(\mathrm{Fa})_{2} \mathrm{PF}_{6}$. We consider a Q1D system with a partially filled conduction band undergoing a Peierls transition. The system is then characterized by the occurrence of an energy gap with a quali-

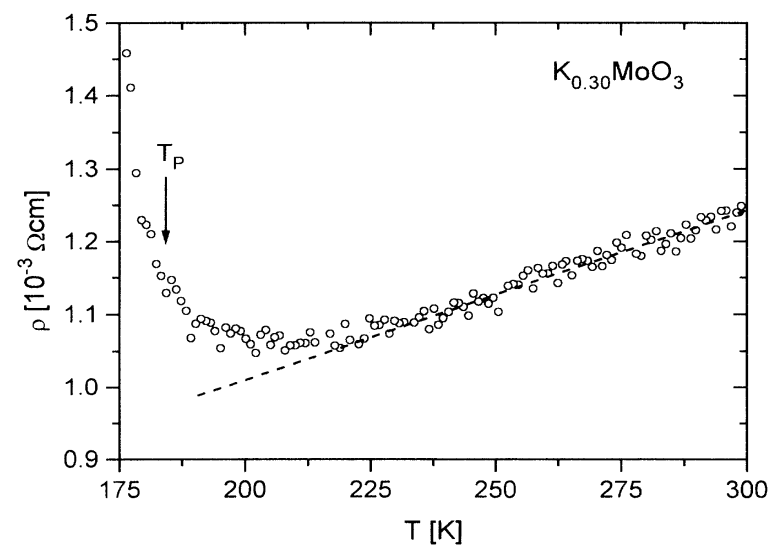

FIG. 2. Temperature dependence of the resistivity in the blue bronze above the Peierls transition. The dashed line indicates a linear temperature dependence above $225 \mathrm{~K}$, which is known from conventional metals at high temperatures. 


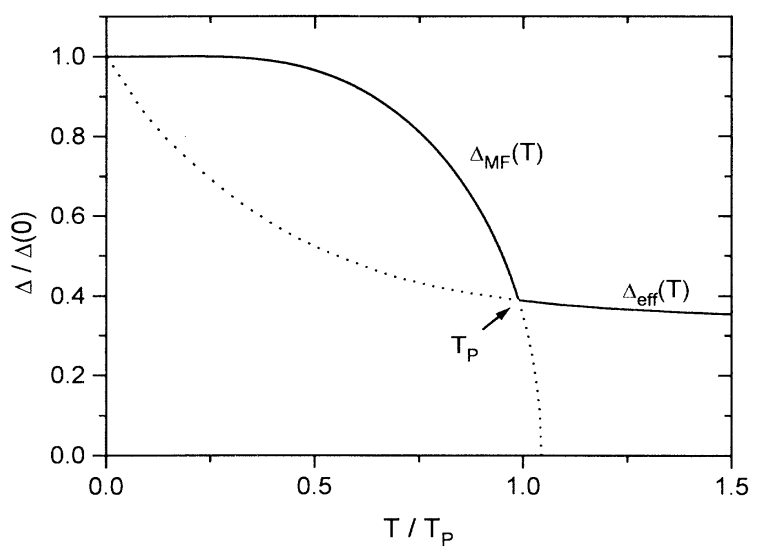

FIG. 3. Schematic temperature dependence of the energy gap in Q1D conductors with a Peierls transition to a CDW ground state. Below $T_{P}$ the gap follows a scaled mean-field dependence (Ref. 25); above $T_{P}$ a pseudogap (often called an effective gap) (Ref. 26) is used.

tative temperature dependence as described above. However, the exact temperature dependence of the gap $2 \Delta(T)$ below and above the transition is unknown and should be determined by analyzing the conductivity data. The description of the conductivity is based on the following assumptions. (a) The thermally activated electrons and holes in the bands above and below the Peierls gap or pseudogap are dominant. (b) Counterions give rise to the partial filling of the unstable metallic conduction band, but due to their spatial separation from the $1 \mathrm{D}$ chains they do not create additional states in the Peierls gap or pseudogap. Also, their contribution to scattering is negligible. ${ }^{27}$ (c) There is quasiclassical transport described by the Boltzmann equation. (d) Further, the relaxation time approximation is used with an energy and temperature-dependent relaxation time $\tau(\varepsilon, T)$. The validity of the Boltzmann description implies the condition $\bar{\tau} \gg \hbar / \bar{\varepsilon}$ for the averaged relaxation time $\bar{\tau}$ and energy $\bar{\varepsilon}$ at the temperature $T$. The conductivity due to both electrons and holes (for a band structure symmetric with respect to the midgap energy; see below) is then given by

$$
\sigma=2 e^{2} \mathcal{N} \int_{0}^{\infty} d \varepsilon \mathcal{D}(\varepsilon) \tau(\varepsilon) v^{2}(\varepsilon)\left(-\frac{\partial f^{0}}{\partial \varepsilon}\right) .
$$

Here $\mathcal{N}$ is the number of Q1D chains per unit area, the energy zero is the band edge, and $f^{0}$ is the Fermi-Dirac distribution. The quasiclassical velocity and the $1 \mathrm{D}$ density of states $\mathcal{D}$ (spin degeneration included) are

$$
v(\varepsilon)=\frac{1}{\hbar} \frac{\partial \varepsilon}{\partial k}, \quad \mathcal{D}(\varepsilon)=\frac{2}{\hbar \pi|v(\varepsilon)|},
$$

respectively. Near the Fermi energy $E_{F}$ of the unstable metallic band with an arbitrary band filling the 1D band structure $E_{q}^{(0)}=E_{F} \pm \hbar v_{F} q$ is approximated as a linear function of $q=k-k_{F}$ (with the Fermi wave number $k_{F}$ ). This leads, in the lowest order of perturbation theory, to the band structure $E_{q}=E_{F} \pm \sqrt{\Delta^{2}+\left(\hbar v_{F} q\right)^{2}}$ with a gap $2 \Delta=2 \Delta(T)$, which is symmetric with respect to the midgap energy $E_{F}$. Relative to the band edge (of the upper band) $E_{0}=E_{F}+\Delta$, this is written as

$$
\begin{aligned}
\varepsilon_{q} & =E_{q}-E_{0}=-\Delta+\sqrt{\Delta^{2}+\left(\hbar v_{F} q\right)^{2}}, \\
v(\varepsilon) & =v_{F} \frac{\sqrt{\varepsilon^{2}+2 \Delta \varepsilon}}{\varepsilon+\Delta}
\end{aligned}
$$

with the velocity required in calculating the conductivity [Eq. (1)]. For energies $\varepsilon \ll(\sqrt{2}-1) \Delta$, this means for $k_{B} T \ll 0.4 \Delta$, one can use for Eq. (3) the parabolic approximation as before ${ }^{19}$

$$
\varepsilon_{q} \approx \frac{\hbar^{2} q^{2}}{2 m^{*}}, \quad \frac{1}{m^{*}}=\frac{v_{F}^{2}}{\Delta}, \quad v(\varepsilon)=v_{F} \sqrt{\frac{2 \varepsilon}{\Delta}} .
$$

The band structure and hence the velocity are both, in Eqs. (3) and (4), determined by the two parameters $\Delta$ and $v_{F}$ only. Already in the approximation (3) the band filling and the magnitude of the Fermi wave number do not occur explicitly. The parameter $v_{F}$, the Fermi velocity of the corresponding metallic system, can be determined from optical data. This procedure requires some comment. In the systems we consider here, the Peierls gap or pseudogap is small $(<200 \mathrm{meV})$. Optically observed interband transitions have an energy larger than $3 \mathrm{eV}^{28}$ But in spite of the existence of this small gap, even in the temperature region below the Peierls transition, there occurs a well pronounced optical reflectivity edge at about $1.5 \mathrm{eV}$, leading to a sharp plasmon peak in the energy loss function. This plasmon peak is influenced only a little by the small pseudogap at room temperature and thus one can describe the optical data with a Drude model including several higher lying interband transitions. ${ }^{28}$ Then one can connect the resulting plasmon frequency $\omega_{p}$ with the Fermi velocity of the corresponding metallic system $v_{F}$. For a $1 \mathrm{D}$ system one has $\omega_{p}^{2}=\left.\left(2 e^{2} \mathcal{N} / \epsilon_{\infty} \epsilon_{0} \hbar^{2} \pi\right)(\partial \varepsilon / \partial k)\right|_{k_{F}}\left(\epsilon_{\infty}=1\right.$ if the interband transitions are taken into account explicitly in the fit of the optical data; otherwise $\epsilon_{\infty}$ is the background dielectric constant). Thus we use in the following

$$
\hbar v_{F}=\frac{\left(\hbar \omega_{p}\right)^{2} \pi \epsilon_{\infty} \epsilon_{0}}{2 e^{2} \mathcal{N}}
$$

in the band structure expressions (3) and (4). The necessary parameters for the three systems are given in Table II.

Finally, one needs an approximation for the relaxation time in Eq. (1). Since scattering by charged counterions is expected to give a negligible contribution, ${ }^{27}$ there remains the scattering by phonons. Acoustical deformation potential scattering gives, in the $1 \mathrm{D}$ case,${ }^{19}$

$$
\tau(\varepsilon)=\frac{\mathcal{B}}{\mathcal{D}(\varepsilon) T}, \quad \mathcal{B}=\frac{\hbar M c_{s}^{2}}{k_{B} 2 \pi a E_{a}^{2}},
$$

where $M$ is the molecular mass, $c_{s}$ the velocity of sound, $a$ the lattice constant in chain direction, and $E_{a}$ the acoustical deformation potential. The dependence $(6)$ is valid for phonon energies $\hbar \omega \ll k_{B} T$. Its application at lower temperatures is therefore only possible if the velocity of sound is relatively small, which is the case for the mate- 
TABLE II. Material parameters derived from optical reflectivity measurements and crystal structure analysis, and the Fermi velocity and energy calculated from them. The lattice constant $a$ represents in all three systems the lattice constant along the highly conducting crystal axis (due to different crystallograhic space groups, a different nomenclature can be found in the literature for the inorganic systems).

\begin{tabular}{lccc}
\hline \hline Parameter & $(\mathrm{Fa})_{2} \mathrm{PF}_{6}$ & $\left(\mathrm{TaSe}_{4}\right)_{2} \mathrm{I}$ & $\mathrm{K}_{0.30} \mathrm{MoO}_{3}$ \\
\hline$\hbar \omega_{p}(\mathrm{eV})$ & $1.5^{\mathrm{a}}$ & $1.2^{\mathrm{b}}$ & $2.7^{\mathrm{c}}$ \\
$\epsilon_{\infty}$ & 1 & 7.6 & 1 \\
$a(\AA)$ & 6.61 & 12.76 & 7.55 \\
$\mathcal{N}\left(\AA^{-2}\right)$ & 0.011 & 0.022 & 0.025 \\
$v_{F}(\mathrm{~cm} / \mathrm{s})$ & $2.65 \times 10^{7}$ & $6.59 \times 10^{7}$ & $3.83 \times 10^{7}$ \\
$E_{F}(\mathrm{eV})$ & 0.55 & 0.6 & 0.6 \\
\hline \hline
\end{tabular}

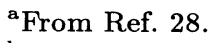

${ }^{b}$ From Ref. 29.

${ }^{\mathrm{c}}$ From Ref. 30.

rials we consider here. With Eqs. (6), (2), and (3) the conductivity (1) becomes

$$
\sigma(T)=\frac{C}{2 k_{B} T} \int_{0}^{\infty} \frac{x^{2}+2 x y}{(x+y)^{2}} \frac{e^{x+y}}{\left(e^{x+y}+1\right)^{2}} d x
$$

with $x=\varepsilon / k_{B} T$ and $y=\Delta(T) / k_{B} T$, where the constant $C$

$$
C=\frac{2 e^{2} \mathcal{N} M c_{s}^{2}}{\pi \hbar a}\left(\frac{\hbar v_{F}}{E_{a}}\right)^{2}
$$

contains $v_{F}$ from Eq. (5) and does not depend on the detailed model for the band structure and the filling of the band, which is different in the three groups of materials investigated here. The equation for the conductivity [Eq. (7)] contains only the temperature-dependent gap and one constant $C$. It is well suited to determine the temperature dependence of the gap from the measured conductivity data. This is more easily done if the parabolic approximation (4) can be applied. Then one can evaluate Eq. (7) analytically and obtain the expression

$$
\sigma(T)=C \frac{\ln \left(1+e^{-\Delta(T) / k_{B} T}\right)}{\Delta(T)},
$$

used in our previous work ${ }^{19}$ with a connection between $v_{F}$ in the constant $C$ [Eq. (8)] and the Fermi energy $\left(v_{F}=\right.$ $\left.a E_{F} / 2 \hbar\right)$ in a special model for the band structure. We further mention that for a vanishing gap $\Delta \equiv 0$, Eq. (7) yields

$$
\sigma=\frac{C}{4 k_{B} T}=e^{2} \mathcal{N} \mathcal{D}_{F} \tau_{F} v_{F}^{2} .
$$

This is the same linear temperature dependence as for a conventional metal at higher temperatures caused by deformation potential scattering. Moreover, similar to Ref. 19 also for the more general expression (7), one can express the mean mobility, the mean free path, and the mean relaxation time through the measured values of the conductivity as functions of $T$ (see the Appendix).
So far only acoustical deformation potential scattering has been taken into account. The approximation (6) requires the phonon energy to be small compared to the temperature. Indeed, in $(\mathrm{Fa})_{2} \mathrm{PF}_{6}$ our analysis led to an estimation of some $\mathrm{meV}$ for the zone edge energy of the involved acoustical phonons. For intermediate phonon energies a detailed knowledge of the phonon spectrum would be required. Nevertheless, one can estimate the contribution of higher energetic nonpolar optical phonons with $\hbar \omega_{\text {opt }} \gg k_{B} T$. Similar to Ref. 27 , one obtains

$$
\tau_{\mathrm{opt}}(\varepsilon)=\frac{1}{\mathcal{D}(\varepsilon)} \frac{M \omega_{\mathrm{opt}}}{4 \pi a \alpha^{2}} e^{\hbar \omega_{\mathrm{opt}} / k_{B} T}
$$

where $\alpha$ is the electron-phonon coupling constant (in the case of organic $1 \mathrm{D}$ systems with a conjugated $\pi$-electron system about $4 \mathrm{eV} / \AA$ ). The total relaxation time is then determined by the smaller one according to $\tau_{\text {tot }}^{-1}=\tau^{-1}+$ $\tau_{\text {opt }}^{-1}$. To compare Eqs. (6) and (11) they are conveniently written in the following dimensionless form (with $T_{r}=$ $300 \mathrm{~K})$ :

$$
\begin{aligned}
\tilde{\tau} & \equiv \tau \mathcal{D}(\varepsilon) \frac{T_{r}}{\mathcal{B}}=\frac{T_{r}}{T}, \\
\tilde{\tau}_{\mathrm{opt}} & \equiv \tau_{\mathrm{opt}} \mathcal{D}(\varepsilon) \frac{T_{r}}{\mathcal{B}}=\gamma e^{\hbar \omega_{\mathrm{opt}} / k_{B} T}, \\
\gamma & =\frac{\pi^{2}}{2}\left(\frac{k_{B} T_{r}}{\hbar \omega_{\mathrm{ac}}}\right)\left(\frac{\omega_{\mathrm{opt}}}{\omega_{\mathrm{ac}}}\right)\left(\frac{E_{a}}{\alpha a}\right)^{2},
\end{aligned}
$$

where in the constant $\gamma$ the approximate acoustical phonon zone edge frequency is $\omega_{\mathrm{ac}}=c_{s}(\pi / a)$. Due to the exponential in Eq. (13), $\tilde{\tau}_{\text {opt }}$ decreases much faster with increasing temperature than $\tilde{\tau}$ in Eq. (12). Thus in the region between 50 and $300 \mathrm{~K}$ one has, e.g., for $\hbar \omega_{\text {opt }} \simeq 3 k_{B} T_{r}$ and $\gamma>1$ a much larger $\tilde{\tau}_{\text {opt }}$ than $\tilde{\tau}$ (and hence $\tilde{\tau}_{\text {opt }}$ negligible), but $\tilde{\tau}_{\text {opt }}$ becomes important near room temperature for $\gamma<0.5$, as shown in Fig. 4 .

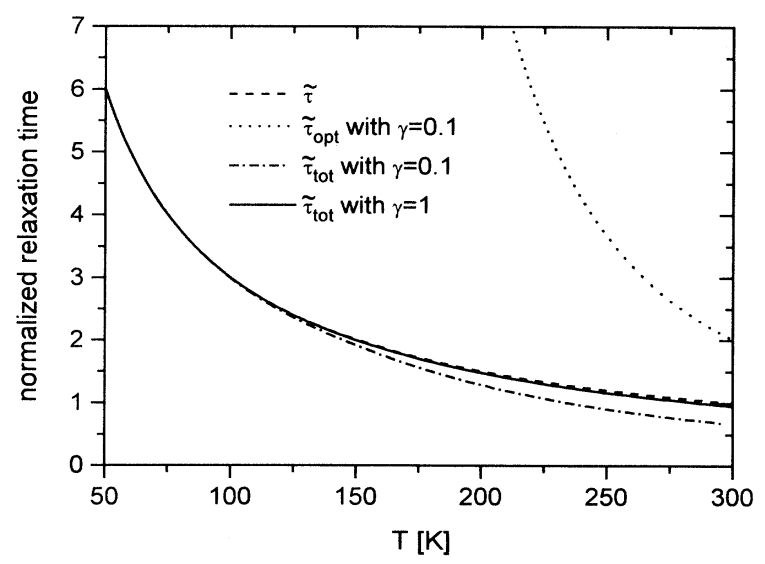

FIG. 4. Temperature dependence of the normalized relaxation times given in Eqs. (12)-(14). Dashed line, $\tilde{\tau}$ following from acoustical deformation potential scattering; dotted line, $\tilde{\tau}_{\text {opt }}$ following from higher energetic optical phonon scattering with $\hbar \omega_{\text {opt }}=3 k_{B} T_{r}$ and $\gamma=0.1$; the resulting total relaxation time $\tilde{\tau}$ is shown for $\gamma=0.1$ (full line) and for $\gamma=1$ (dash-dotted line). For $\gamma=1, \tilde{\tau}_{\text {opt }}$ is so large that its contribution is negligible. 


\section{RESULTS}

In Ref. 19 we applied the theory to $(\mathrm{Fa})_{2} \mathrm{PF}_{6}$ using the parabolic approximation (4) leading to the analytical expression (9) for the conductivity. The experimental data were described well with the gap shown in Fig. 3 and only one parameter, the constant $C$ in Eq. (9) [apart from $\Delta(0)$ and $\left.T_{P}\right]$. Using this fit parameter and Eq. (8) we estimated [with $v_{F}=a E_{F} / 2 \hbar$ and $E_{a}=(2 / 3) E_{F}$ ] the sound velocity $\left(c_{s} \approx 310 \mathrm{~m} / \mathrm{s}\right)$ and the zone edge acoustical phonon energy $\left(\hbar \omega_{\text {ac }} \approx 1 \mathrm{meV}\right)$. Both values were found to be in a reasonable range.

With the full theory [Eq. (7)] the experimental data of the conductivity of all three systems are described almost perfectly using the energy gap as shown in Fig. 3 . In the logarithmic plot of Fig. 1 the deviations between the experimental data and the fit are more or less within the graphic linewidth. These results indicate that indeed the charge transport is dominated by thermally activated electrons and holes in the bands above and below the Peierls gap (or pseudogap above $T_{P}$ ) and by acoustical deformation potential scattering. On the other hand, such a fit of the conductivity data has some disadvantages. At first, for a real system the Peierls gap below the transition temperature will deviate from the simple mean-field dependence used in Ref. 19 due to several effects such as three dimensionality, fluctuations, electronelectron interactions, deviations from a free-electron-like band structure, and band filling different from $1 / 2$. Further, reliable values for the pseudogap above $T_{P}$ are not generally available and, in addition, one has to expect a difference between the fluctuating pseudogap as determined from the magnetic susceptibility as a static property and a transport gap connected with mobility edges.

Thus, as proposed in Ref. 19, we will now proceed in the inverse manner and use the theory to determine the whole temperature dependence of the gap from the measured conductivities. But contrary to the previous work, the formulation given in Sec. III does not depend on special assumptions on the band structure of the unstable metallic conduction band and on its filling. Moreover, for the system with the gap the more general expression for the velocity [Eq. (3)] is used leading to the expression (7) for the conductivity and in this way the description is also valid if the gap becomes comparable to or even less than $k_{B} T$. Since both $\Delta(T)$ and the constant $C$ have to be determined via Eq. (7) from the measured conductivity data, one needs in addition the value of the gap at one temperature. If available, such a value can be taken, e.g., from optical or paramagnetic spin susceptibility data. Here we use the fact that for all three substances the conductivity shows, in the region $50 \mathrm{~K}$ $<T<100 \mathrm{~K}$, roughly a thermally activated behavior with a constant activation energy. Indeed, for sufficiently small $T$, Eq. (7) is reduced to Eq. (9) and the latter to $\sigma(T) \approx(C / \Delta) \exp \left\{-\Delta / k_{B} T\right\}$, allowing for the direct determination of the ground-state energy gap, which will be denoted here as $2 \Delta(0)$ (the deviation of the conductivity from the activated behavior below $50 \mathrm{~K}$ was mentioned already in Sec. II). The values of $\Delta(0)$ used for the samples considered here are given in Table III. With the
TABLE III. Parameters following from the quantitative analysis for the three representatives, which are shown in Figs. 1 , and 5-7. For comparison, values of the sound velocity from neutron scattering are given.

\begin{tabular}{lccc}
\hline \hline Parameter & $(\mathrm{Fa})_{2} \mathrm{PF}_{6}$ & $\left(\mathrm{TaSe}_{4}\right)_{2} \mathrm{I}$ & $\mathrm{K}_{0.30} \mathrm{MoO}_{3}$ \\
\hline$\Delta(0)(\mathrm{meV})$ & 90 & 139 & 41 \\
$C(\mathrm{eV} / \Omega \mathrm{cm})$ & 236 & $1.14 \times 10^{4}$ & 72.1 \\
$M c_{s}^{2}(\mathrm{eV})$ & 0.42 & 3.63 & 0.035 \\
$c_{s}(\mathrm{~m} / \mathrm{s})$ & 449 & 1390 & 188 \\
$M(\mathrm{u})$ & 202 & 181 & 96 \\
$c_{s}^{(\mathrm{ph})}(\mathrm{m} / \mathrm{s})$ & & $3915^{\mathrm{a}}$ & $7540^{\mathrm{b}}$ \\
\hline \hline
\end{tabular}

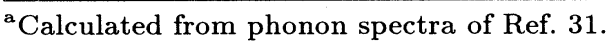

${ }^{\mathrm{b}}$ Calculated from phonon spectra of Ref. 32 .

value of $\Delta$ one fixes also the constant $C$ (Table III). Obviously the assumption of sufficiently low temperature is fulfilled well. Only in the case of $\mathrm{K}_{0.30} \mathrm{MoO}_{3}$ is there a small uncertainty in the determination of $\Delta(0)$, which is only four to five times larger than $k_{B} T$ between 50 and $100 \mathrm{~K}$.

The results for the temperature dependence of the gap in the entire temperature range are shown in Figs. 5(a)$5(\mathrm{c})$. For $(\mathrm{Fa})_{2} \mathrm{PF}_{6}$ we compare in Fig. 5(a) three curves. The first one (full line) (already shown in Fig. 3) was used in Ref. 19 to fit the data and shows below $T_{P}$ the scaled mean-field dependence and above $T_{P}$ the one following from paramagnetic spin susceptibility. The second curve (dashed line) was determined also in Ref. 19 from the conductivity by using Eq. (9) and $\Delta(60 \mathrm{~K})=90 \mathrm{meV}$. For the third one (dotted line) we used the improved expression (7) and the same value for $\Delta(60 \mathrm{~K})$. This value is so large that the difference between both curves is small for lower temperatures. However, with increasing temperature and decreasing gap, the nonparabolicity of the bands becomes important and the gap determined with the full expression (7) decreases faster and is at room temperature about $15 \mathrm{meV}$ smaller than the gap determined from Eq. (9). Also, it now coincides almost exactly with the values from paramagnetic spin susceptibility. ${ }^{16}$ At the same time, with the full expression (7) the transition from the Peierls gap to the pseudogap is less pronounced. We want to mention also that the procedure depends sensitively on the value used for the gap at low temperatures: reducing $\Delta(60 \mathrm{~K})$ from $90 \mathrm{meV}$ to $88 \mathrm{meV}$ results at room temperature in a difference of 10 meV. The whole dependence obtained with Eq. (7) from the conductivity agrees remarkably well with the scaled mean-field gap below the transition and also yields the existence of the pseudogap above the transition. Similar results for the gap were obtained recently from optical investigations. ${ }^{33}$ The analysis of these data leads to the values $\Delta=100,89$, and $45 \mathrm{meV}$ for 10,140 , and 300 $\mathrm{K}$, respectively. These values are about $10 \mathrm{meV}$ larger than in this work, but this can be due to crystals from different batches, where also in the dc conductivity variations of the ground-state gap of this order are observed. Nevertheless, the optical values demonstrate the decrease of the Peierls gap with increasing temperature and the existence of a pseudogap above the Peierls transition.

The gap for $\left(\mathrm{TaSe}_{4}\right)_{2} \mathrm{I}$ is shown in Fig. 5(b). For the 
low temperature activation energy we obtained from the measured conductivity $\Delta(80 \mathrm{~K})=139 \mathrm{meV}$. Both the ground-state gap and the transition temperature are considerably higher than in the other two substances. However, in spite of the large gap, a considerable difference (almost $10 \mathrm{meV}$ at $300 \mathrm{~K}$ ) occurs when calculating the gap from the measured data with the full expression (7) (dashed line) or with the parabolic approximation (9) (full line). Unexpectedly the resulting pseudogap above the transition increases with temperature. This behavior is probably an artifact arising from the oversimplified assumption about the scattering mechanism. As shown in Sec. III, higher energetical phonons can lead to a reduction of the total relaxation time at higher temperatures if the preexponential factor (14) in Eq. (13) becomes small.

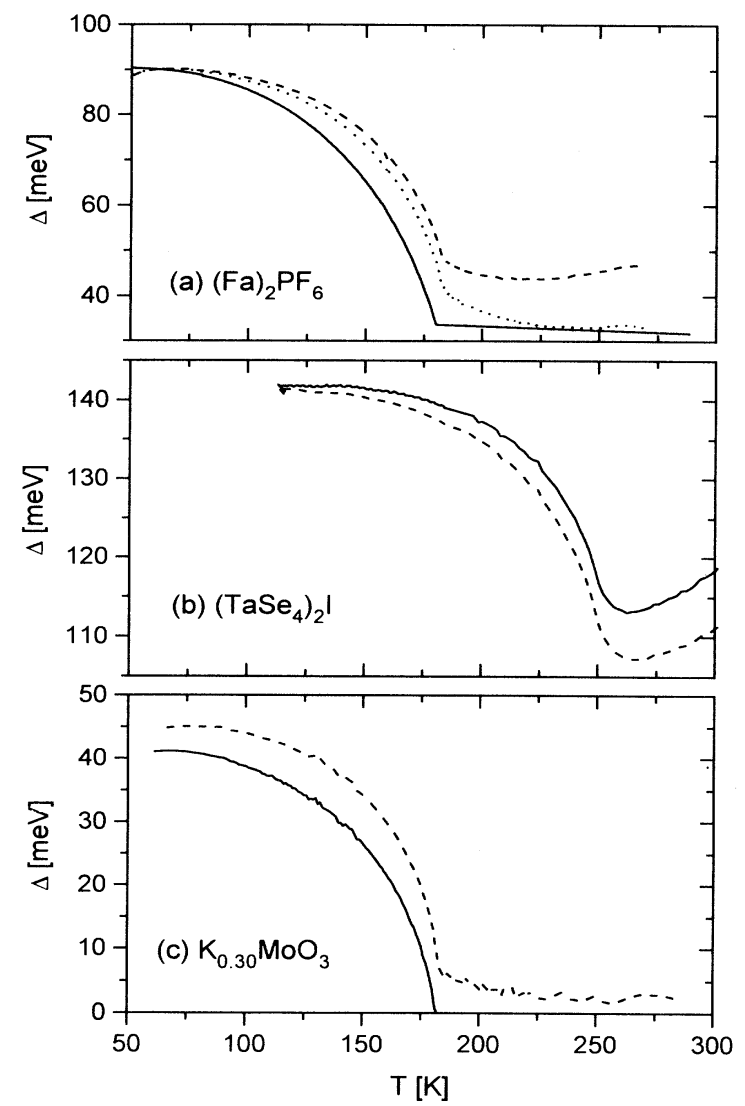

FIG. 5. Temperature dependence of the energy gap in the three systems as derived from dc-conductivity data. (a) $(\mathrm{Fa})_{2} \mathrm{PF}_{6}$ : The full line shows the model dependence given in Fig. 3, the dashed line was calculated with the parabolic band structure approximation using Eq. (9) for the conductivity, and the dotted line shows the gap following from the full theory [Eq. (7)] for the conductivity; $\Delta(60 \mathrm{~K})=90 \mathrm{meV}$ has been used as the value for the low temperature activation energy. (b) $\left(\mathrm{TaSe}_{4}\right)_{2} \mathrm{I}$ : The full line is the gap derived from Eq. (9) and the dashed line is the dependence from the full description [Eq. (7)]; $\Delta(80 \mathrm{~K})=139 \mathrm{meV}$ is used. (c) $\mathrm{K}_{0.30} \mathrm{MoO}_{3}$ : Both curves are derived from the full expression (7); the full line was calculated using the value $\Delta(60 \mathrm{~K})=41 \mathrm{meV}$, following from the low temperature activated behavior, and the dashed line with a value chosen a little larger, e.g., as $\Delta(60 \mathrm{~K})=45 \mathrm{meV}$.
Although Eq. (14) contains several parameters that are unknown or can be estimated only very roughly, the large value of the lattice constant $a$ in chain direction (due to the $45^{\circ}$ rotation of the adjacent $\mathrm{Se}_{4}$ unit) may be indicative for a smaller value of $\gamma$. Also the band structure is more complicated especially due to interchain coupling being responsible for a further splitting of the band and the occurrence of an incommensurate Fermi wave number. ${ }^{34}$ Nevertheless, the existence of a pseudogap of such a magnitude in $\left(\mathrm{TaSe}_{4}\right)_{2} \mathrm{I}$ is confirmed by recent optical conductivity measurements of several authors. ${ }^{17,35}$

In the case of $\mathrm{K}_{0.30} \mathrm{MoO}_{3}$ [Fig. 5(c)] the Peierls transition temperature is similar to the fluoranthene radical cation salts near $180 \mathrm{~K}$; the transition itself is more pronounced. There is another difference that may be seen in the conductivity (Fig. 1) above the transition. There the conductivity decreases slightly with increasing temperature like in a metallic state. As shown in Fig. 2, there is indeed a linear temperature dependence of the resistivity above $225 \mathrm{~K}$ in accordance with Eq. (10). Thus the existence of a residual fluctuating pseudogap above the transition seems to be questionable. Indeed optical investigations ${ }^{18}$ also seem to be consistent with a metallic behavior above $225 \mathrm{~K}$ and an opening of the pseudogap between $225 \mathrm{~K}$ and the transition at $183 \mathrm{~K}$. Our conductivity data lead from the activated low temperature behavior to $\Delta(0)=41 \mathrm{meV}$. With this value the measured conductivity yields with Eq. (7) above the transition temperature a vanishing gap [Fig. 5(c), full line]. On the other hand, as already mentioned above, due to the relatively small value of $\Delta(0)$ its determination is not sufficiently exact. Thus, in Fig. 5(c) we show also the curve that is obtained when the low temperature gap is chosen a little higher, e.g., as $\Delta(0)=45 \mathrm{meV}$ (dashed line). In this case there remains a small pseudogap above the transition. One should mention that the description used here is less reliable in the case of the blue bronze since, contrary to the simple band structure models with a three-quarters filled band, ${ }^{36}$ more sophisticated models lead to a partial occupation of two bands and the metalto-semiconductor transition is connected then with the nesting properties of the two Fermi surfaces. ${ }^{24}$

With the knowledge of the temperature-dependent gap $2 \Delta(T)$ (Fig. 5), the constant $C$ (Table III), and the Fermi velocity $v_{F}$ (Table II) [and, approaching the degenerate limit, the Fermi energy (Table II)] one can determine several conductivity-related quantities using the description given in the Appendix. At first, we determine the density $n$ [Eq. (A4)], which coincides with the actual electron and hole densities in the nondegenerate case $\left(\Delta \gg k_{B} T\right)$, which is approximately fulfilled in $(\mathrm{Fa})_{2} \mathrm{PF}_{6}$ and $\left(\mathrm{TaSe}_{4}\right)_{2} \mathrm{I}$ up to room temperature and for the blue bronze only up to about $160 \mathrm{~K}$ since the gap almost vanishes at $T_{P}$. The density of electrons taking part in the transport is in the degenerate limit proportional to the applied field [see Eq. (A11)] and cannot be represented by an expression such as Eq. (A4). The resulting density $n$ shows the same general behavior as the conductivity (Fig. 1) with an increase of eight to ten orders of magnitude from 50 to $300 \mathrm{~K}$ and reaches values at room temperature of almost $10^{20} \mathrm{~cm}^{-3}$ for $(\mathrm{Fa})_{2} \mathrm{PF}_{6}$ 
and $2 \times 10^{18} \mathrm{~cm}^{-3}$ for $\left(\mathrm{TaSe}_{4}\right)_{2} \mathrm{I}$ [this value is smaller due to a larger gap and also due to the larger $v_{F}(\mathcal{N}$ differs only slightly)]. For $\mathrm{K}_{0.30} \mathrm{MoO}_{3}$ already at $160 \mathrm{~K}$ the density exceeds $5 \times 10^{19} \mathrm{~cm}^{-3}$ since the gap is considerably smaller.

According to Eqs. (A3) and (A9) one obtains, from the measured conductivity, the mobility $\mu=\sigma / 2 e n$ (reasonable for $\Delta>k_{B} T$ ), which is shown in Fig. 6. Due to the similar behavior of the conductivity and the density $n$ this mean mobility varies only by about a factor of 2 over the entire temperature interval. In the case of $(\mathrm{Fa})_{2} \mathrm{PF}_{6}$ and $\left(\mathrm{TaSe}_{4}\right)_{2} \mathrm{I}$ the mobility at first increases with decreasing temperature due to the freeze-out of phonons. However, at the phase transition the formation of the lattice superstructure leads to an additional scattering mechanism, which reduces the mobility abruptly. At temperatures of about $30 \mathrm{~K}$ below the respective Peierls transition the mobility begins to increase again, because at low enough temperatures the opening of the energy gap and therefore the lattice distortion is practically complete and there remains again the phonon contribution. In the case of $\mathrm{K}_{0.30} \mathrm{MoO}_{3}$ the definition of the mobility is applicable only below about $160 \mathrm{~K}$. The magnitude of the mobility shows moderate values in the cases of $(\mathrm{Fa})_{2} \mathrm{PF}_{6}$ and the blue bronze and is rather large for $\left(\mathrm{TaSe}_{4}\right)_{2} \mathrm{I}$. These medium to large values reflect especially the small effec-

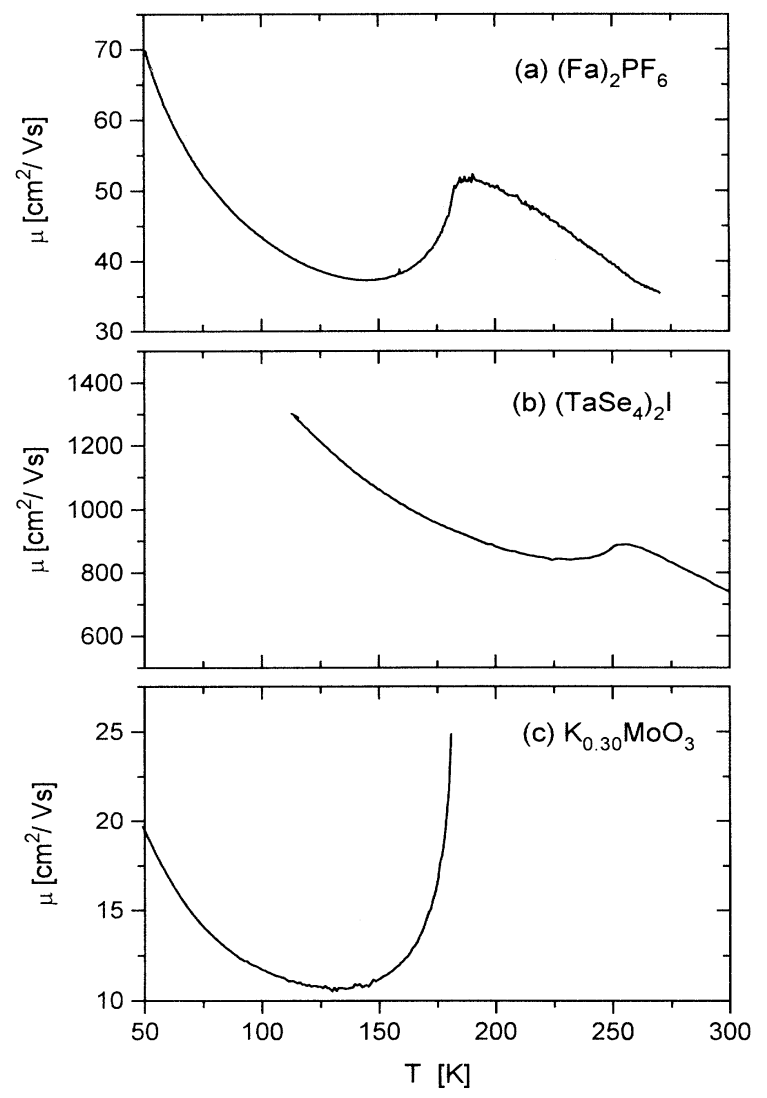

FIG. 6. Temperature dependence of the carrier mobility in the three systems as determined from the experimental conductivity data and the carrier density [Eq. (A4)]. tive mass to be discussed below. On the other hand, they arise from the ineffectivity of scattering by charged counterions due to their spatial separation from the $1 \mathrm{D}$ conduction channel of the chains. While the meaning of the mobility and the density $n$ is restricted to $\Delta \gg k_{B} T$, this is not the case for the mean relaxation time [Eq. (A2)]. It can be obtained from the measured conductivity via Eq. (A3) by calculating additionally the effective transport mass [Eq. (A5)]. The peculiarity of this mass is its dependence on the energy-dependent relaxation time. As before we include here only acoustical deformation potential scattering. The effective transport masses (Fig. 7) are rather small due to the small gap (for the blue bronze) or due to the high Fermi velocity [for $\left(\mathrm{TaSe}_{4}\right)_{2} \mathrm{I}$ with the larger gap]. The temperature dependence of this mass [Eq. (A5)] arises from the deviation of the band structure [Eq. (3)] from the parabolic behavior [Eq. (4)] near the band edges, which becomes important for $\Delta<k_{B} T$. But as seen in Fig. 7 , at $50 \mathrm{~K}$ the effective transport mass deviates from the band edge value $\Delta / v_{F}^{2}$, showing that at these low temperatures, higher energies of the carriers contribute to the mean value. Nevertheless, the total variation of the effective mass over the entire temperature interval is less than $35 \%$ of the low temperature

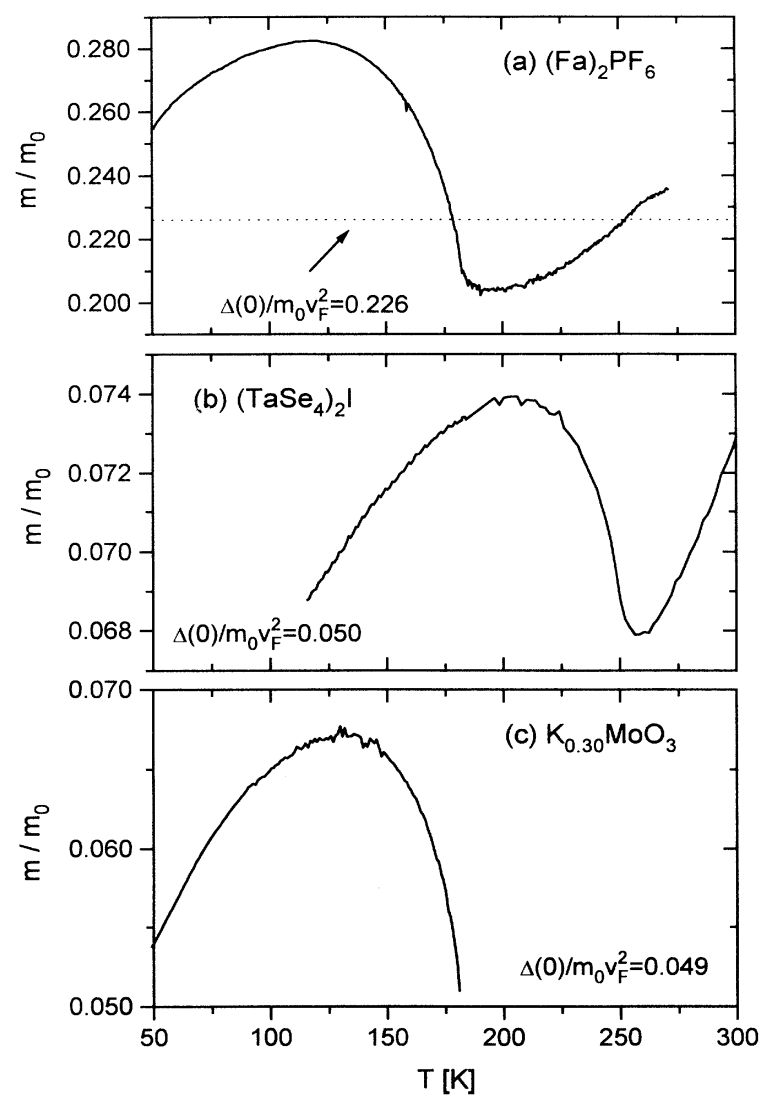

FIG. 7. Temperature dependence of the effective transport mass [Eq. (A5)] of the three systems using the gap derived from conductivity data and Eqs. (2), (3), and (6). The values of $\Delta(0) / m_{0} v_{F}^{2}$ give the low temperature band edge value of the effective transport mass. 
band edge value. Due to this small variation the temperature dependence of the relaxation time [Eq. (A2)] is qualitatively the same as the one of the mobility with a total variation of about a factor of 2 . In addition, the maximum near the transition temperature $T_{P}$ is less pronounced than in the mobility. More important is that, in spite of the large values of the mobility and due to the small effective masses, the relaxation times are small with values of 5-10 fs for $(\mathrm{Fa})_{2} \mathrm{PF}_{6}, 30-70 \mathrm{fs}$ for $\left(\mathrm{TaSe}_{4}\right)_{2} \mathrm{I}$, and $0.4-0.7$ fs for $\mathrm{K}_{0.30} \mathrm{MoO}_{3}$. Similarly, one can calculate with Eq. (A13) the value of $\bar{\tau} \bar{\varepsilon} / \hbar$, which should be large compared to unity for the Boltzmann description to be applicable. However, we obtain the following values: 0.04-0.14 for $(\mathrm{Fa})_{2} \mathrm{PF}_{6}, 0.3-0.7$ for $\left(\mathrm{TaSe}_{4}\right)_{2} \mathrm{I}$, and 0.010.025 for $\mathrm{K}_{0.30} \mathrm{MoO}_{3}$. From these values the applicability of the Boltzmann description seems to be questionable at first sight. But we should mention that these values follow from the experimental conductivities, ascribing the temperature dependence to the dominance of acoustical deformation potential scattering. Thus the numbers obtained depend at first on the absolute values of the conductivity, which in turn depend on geometrical factors which can be determined only with some error. Further, crystal imperfections are expected to prevent part of the chains from effectively contributing to the current. Thus the real values for the constant $C$ determining the contribution of the considered scattering mechanism should be larger, reducing the apparent violation of the condition for the applicability of the Boltzmann equation. Furthermore, usually a formal criterion for the applicability of an approximate theoretical description is much stronger than the practical usage.

Finally, a rough estimate for the velocity of sound will be presented using the fitted constant $C$ (Table III). According to Eq. (8) we need at first the deformation potential constant $E_{a}$. We use here the connection with the Fermi energy $E_{a}=(2 / 3) E_{F}$ (strictly valid only in the 3D electron gas) and the values for the Fermi energy given in Table II. Resulting values for $M c_{s}^{2}$ are given in Table III. To obtain the velocity of sound we additionally need the molecular mass determining the acoustical phonons, which are interacting with the electron system. We assume $M=202 \mathrm{u}\left(\mathrm{C}_{16} \mathrm{H}_{10}\right.$ molecule $)$ for $(\mathrm{Fa})_{2} \mathrm{PF}_{6}$, $M=181 \mathrm{u}$ (Ta atom) for $\left(\mathrm{TaSe}_{4}\right)_{2} \mathrm{I}$, and $M=96 \mathrm{u}$ (Mo atom) for the blue bronze. The resulting velocity of sound is compared in Table III with values from neutron scattering data. ${ }^{31,32}$ Considering the already mentioned uncertainties in the absolute values of the conductivity, the values obtained for the velocity of sound seem to be acceptable.

\section{CONCLUSION}

We have developed a model for the dc conductivity in organic and inorganic CDW conductors, that allows for the determination of the temperature dependence of the energy gap in the entire temperature range below and above the Peierls transition directly from dc-conductivity data. Our measurements confirm that the same conduction mechanism is responsible for the temperature dependence of the conductivity in the organic radical cation salt $(\mathrm{Fa})_{2} \mathrm{PF}_{6}$, the blue bronze $\mathrm{K}_{0.30} \mathrm{MoO}_{3}$, and the transition-metal compound $\left(\mathrm{TaSe}_{4}\right)_{2} \mathrm{I}$. In all three cases the calculated temperature dependence of the energy gap shows the typical behavior of a Q1D conductor with a Peierls transition to a CDW ground state. Below $T_{P}$ the Peierls gap follows a scaled mean-field dependence; above the Peierls transition up to the mean-field transition temperature $T_{P}^{\mathrm{MF}}$ there remains a pseudogap due to CDW fluctuations. However, due to different degree of one dimensionality and ground-state energy gaps $2 \Delta(0)$ (resulting in different values for $T_{P}^{\mathrm{MF}}$ ), the magnitude and the temperature dependence of the pseudogap above $T_{P}$ show considerable differences between the three systems. In the case of $\left(\mathrm{TaSe}_{4}\right)_{2} \mathrm{I}$ the pseudogap above the transition increases apparently with temperature, which can be attributed to the neglect of nonpolar optical phonon scattering. On the other hand, for $\mathrm{K}_{0.30} \mathrm{MoO}_{3}$ the gap soon vanishes above the Peierls transition and the resistivity becomes proportional to $T$ in accordance with deformation potential scattering in a $1 \mathrm{D}$ metal.

Our results for the temperature dependence and the magnitude of the energy gap in these three types of CDW systems are in good agreement with recent optical reflectivity measurements in the far infrared by other groups. Furthermore, these results demonstrate the importance of fluctuation effects at the Peierls transition in Q1D conductors.

\section{ACKNOWLEDGMENTS}

We thank M. Schwoerer and D. Berner (Karlsruhe) for helpful discussions and J. Gmeiner for growing the $(\mathrm{Fa})_{2} \mathrm{PF}_{6}$ crystals. We are indebted to J. Dumas (Grenoble) for providing us with some samples of $\mathrm{K}_{0.30} \mathrm{MoO}_{3}$ and P. Monceau (Grenoble) for the $\left(\mathrm{TaSe}_{4}\right)_{2} \mathrm{I}$ crystals. This work was funded by the Fonds der Chemischen Industrie and BASF/BMFT.

\section{APPENDIX: CONDUCTIVITY-RELATED QUANTITIES}

The band structure [Eq. (3)] becomes parabolic [Eq. (4)] for $\Delta \gg k_{B} T$. On the other hand, Eq. (3) contains, in the limit $\Delta \rightarrow 0$, the linear dispersion relation near the Fermi energy. At the same time one has, from $\Delta \gg k_{B} T$ to $\Delta \rightarrow 0$, the transition from nondegenerate to degenerate statistics. The general expression (7) for the conductivity contains both limits correctly. But in order to determine conductivity-related quantities one has to generalize the usual expressions ${ }^{19}$ appropriately. Defining the weighted mean relaxation time as (integration over one of the two symmetric bands)

$$
\bar{\tau}=\frac{\int d \varepsilon \mathcal{D} \tau \varepsilon\left(-\partial f^{0} / \partial \varepsilon\right)}{\int d \varepsilon \mathcal{D} \varepsilon\left(-\partial f^{0} / \partial \varepsilon\right)},
$$

one obtains correctly the nondegenerate and degenerate limits 
$\bar{\tau} \longrightarrow \begin{cases}\frac{\int d \varepsilon \mathcal{D} \tau \varepsilon f^{0}}{\int d \varepsilon \mathcal{D} \varepsilon f^{0}}=\frac{\int d \varepsilon \mathcal{D} \tau\left(\varepsilon / \frac{1}{2} k_{B} T\right) f^{0}}{\int d \varepsilon \mathcal{D} f^{0}} & \text { (nondegenerate) } \\ \tau_{F} & \text { (degenerate) }\end{cases}$

where $\tau_{F}$ is the value of the relaxation time at the Fermi energy for $\Delta \equiv 0$. In order to express the conductivity in the form common for the nondegenerate case

$$
\sigma=2 \frac{e^{2} n \bar{\tau}}{m^{*}}
$$

(the factor 2 is due to electron and hole contributions), because of Eq. (A1) one has to define the density and the weighted effective transport mass as

$$
n=\mathcal{N} \int d \varepsilon \mathcal{D} 2 \varepsilon\left(-\partial f^{0} / \partial \varepsilon\right)
$$

and

$$
\frac{1}{m^{*}}=\frac{\int d \varepsilon \mathcal{D} \tau v^{2}\left(-\partial f^{0} / \partial \varepsilon\right)}{\int d \varepsilon \mathcal{D} \tau 2 \varepsilon\left(-\partial f^{0} / \partial \varepsilon\right)}
$$

Indeed, in the nondegenerate limit Eq. (A4) becomes the usual density

$$
n \longrightarrow \mathcal{N} \int d \varepsilon \mathcal{D} \frac{\varepsilon}{\frac{1}{2} k_{B} T} f^{0}=\mathcal{N} \int d \varepsilon \mathcal{D} f^{0}
$$

In the same limit Eq. (A5) becomes [using partial integration and the generally valid relation $\tau(\varepsilon) \sim \mathcal{D}^{-1}(\varepsilon)$; see Eq. (6) or (11)]

$$
\frac{1}{m^{*}} \longrightarrow \frac{\int d \varepsilon \mathcal{D} \tau v \frac{d v}{d \varepsilon} f^{0}}{\int d \varepsilon \mathcal{D} \tau f^{0}}
$$

Note that Eq. (A7) contains the quasiclassical mass according to

$$
\frac{1}{\hbar^{2}} \frac{\partial^{2} \varepsilon}{\partial q^{2}}=\frac{1}{\hbar} \frac{\partial}{\partial q} v=\frac{1}{\hbar} \frac{\partial \varepsilon}{\partial q} \frac{\partial v}{\partial \varepsilon}=v \frac{\partial v}{\partial \varepsilon} .
$$

The peculiarity of the transport mass [Eq. (A5)] is the weight function $\tau(\varepsilon)$ in the mean value. Equation (A3) is written formally as in the nondegenerate case. Indeed, in this case the current density is

$$
j=\sigma E=2 e n \delta v \text { with } \delta v=\mu E, \mu=\frac{e}{m^{*}} \bar{\tau}
$$

where the mobility $\mu$ is the mean excess (drift) velocity per unit field of all carriers.

Formally one can calculate also Eqs. (A4), (A5), and $e \bar{\tau} / m^{*}$ in the degenerate case, yielding

$$
n \longrightarrow 2 \ln 2 \mathcal{N D}_{F} k_{B} T, \frac{1}{m^{*}} \longrightarrow \frac{1}{4 \ln 2} \frac{v_{F}^{2}}{k_{B} T},
$$

where $\mathcal{D}_{F}$ is given by Eq. (2) with $v=v_{F}$. Indeed, in this case the current density is then

$$
j=\sigma E=e \delta n v_{F} \quad \text { with } \quad \begin{aligned}
\delta n & =\mathcal{N} \mathcal{D}_{F} \delta \varepsilon \\
\delta \varepsilon & =v_{F} \text { e } \tau_{F} E
\end{aligned}
$$

where all carriers contributing to the current have the Fermi velocity $v_{F}$. Therefore it becomes meaningless to define a mobility, since now it is the density $\delta n$ of the drift-current carriers, that is proportional to the applied field due to the shift $\delta \varepsilon$ of the Fermi distribution. Thus Eq. (A3) is also valid in the degenerate case with Eqs. (A1), (A4), and (A5). But for the transport in the degenerate case only the mean relaxation time [Eqs. (A1) and (A2)] and the combination

$$
\frac{n}{m^{*}} \longrightarrow \frac{1}{2} \mathcal{N} \mathcal{D}_{F} v_{F}^{2}
$$

remain reasonable. For the given energy dependence of the relaxation time one can calculate the effective mass [Eq. (A5)] and hence one can determine both the mean relaxation time and the mobility from the experimentally determined conductivity data using Eqs. (A3) and (A9). Further, with the mean velocity and the mean energy of the carriers contributing to the current

$$
\bar{v}=\frac{\int d \varepsilon \mathcal{D} v\left(-\partial f^{0} / \partial \varepsilon\right)}{\int d \varepsilon \mathcal{D}\left(-\partial f^{0} / \partial \varepsilon\right)}, \quad \bar{\varepsilon}=\frac{\int d \varepsilon \mathcal{D} \varepsilon\left(-\partial f^{0} / \partial \varepsilon\right)}{\int d \varepsilon \mathcal{D}\left(-\partial f^{0} / \partial \varepsilon\right)}
$$

one obtains then the mean free path $\bar{\lambda}=\bar{\tau} \bar{v}$ and finally one can check whether or not the fundamental criterion $\bar{\varepsilon} \bar{\tau} / \hbar \gg 1$ for the applicability of the Boltzmann transport description is fulfilled.
${ }^{1}$ G. Grüner, Rev. Mod. Phys. 60, 1129 (1988).

2 Charge Density Waves in Solids, edited by L. P. Gorkov and G. Grüner (North-Holland, Amsterdam, 1989).

${ }^{3} \mathrm{~K}$. Bechgaard and D. Jérome, Phys. Scr. T39, 37 (1991).

${ }^{4}$ G. Grüner, Rev. Mod. Phys. 66, 1 (1994).

${ }^{5}$ Electronic Properties of Inorganic Quasi-One-Dimensional Compounds I+II, edited by P. Monceau (Reidel, Dordrecht, 1985).

${ }^{6}$ Low-Dimensional Electronic Properties of Molybdenum Bronzes and Oxides, edited by C. Schlenker (Kluwer, Dordrecht, 1989).
${ }^{7}$ W. Rieß and W. Brütting, Phys. Scr. T49, 721 (1993).

${ }^{8} \mathrm{~W}$. Brütting and W. Rieß, Acta Phys. Polon. A (to be published).

${ }^{9}$ E. B. Lopes, M. J. Matos, R. T. Henriques, M. Almeida, and J. Dumas, Europhys. Lett. 27, 241 (1994).

${ }^{10}$ P. A. Lee, T. M. Rice, and P. W. Anderson, Phys. Rev. Lett. 31, 462 (1973).

${ }^{11}$ M. J. Rice and S. Strässler, Solid State Commun. 13, 125 (1973).

12 J. B. Nielsen and K. Carneiro, Solid State Commun. 33, 1097 (1980). 
13 J. P. Pouget and R. Comes, in Charge Density Waves in Solids (Ref. 2), p. 85.

${ }^{14}$ V. Ilakovac, S. Ravy, J. P. Pouget, W. Rieß, W. Brütting, and M. Schwoerer, J. Phys. (France) IV 3, C2-137 (1993).

${ }^{15}$ D. C. Johnston, M. Maki, and G. Grüner, Solid State Commun. 53, 5 (1985).

${ }^{16}$ U. Köbler, J. Gmeiner, and E. Dormann, J. Magn. Magn. Mater. 69, 189 (1987).

${ }^{17}$ D. Berner, G. Scheiber, A. Gaymann, H. P. Geserich, P. Monceau, and F. Levy, J. Phys. (France) IV 3, C2-255 (1993).

${ }^{18}$ L. Degiorgi, G. Grüner, K. Kim, R. H. McKenzie, and P. Wachter, Phys. Rev. B 49, 14754 (1994).

${ }^{19}$ P. H. Nguyen, G. Paasch, W. Brütting, and W. Rieß, Phys. Rev. B 49, 5172 (1994).

${ }^{20}$ W. Brütting, W. Rieß, and M. Schwoerer, Ann. Phys. (Leipzig) 1, 409 (1992).

${ }^{21}$ L. F. Schneemeyer, F. J. DiSalvo, S. E. Spengler, and J. V. Waszczak, Phys. Rev. B 30, 4297 (1984).

22 Z. Z. Wang, M. C. Saint-Lager, P. Monceau, M. Renard, P. Gressier, A. Meerschaut, L. Guemas, and J. Rouxel, Solid State Commun. 46, 325 (1983).

${ }^{23}$ Crystal Chemistry and Properties of Materials with QuasiOne-Dimensional Structures, edited by J. Rouxel (Reidel, Dordrecht, 1986).

${ }^{24}$ M.-H. Whangbo and L. F. Schneemeyer, Inorg. Chem. 25, 2424 (1986)
${ }^{25}$ H. J. Schulz, in Low-Dimensional Conductors and Superconductors, edited by D. Jérome and L. G. Caron (Plenum, New York, 1985), p. 95.

${ }^{26}$ D. C. Johnston, J. P. Stokes, and R. A. Klemm, J. Magn. Magn. Mater. 54, 1317 (1986).

${ }^{27}$ S. Kivelson and A. J. Heeger, Synth. Met. 22, 371 (1988).

${ }^{28}$ T. Schimmel, B. Koch, H. P. Geserich, and M. Schwoerer, Synth. Met. 33, 311 (1989).

${ }^{29}$ H. P. Geserich, G. Scheiber, F. Levy, P. Gressier, and P. Monceau, Mol. Cryst. Liq. Cryst. 121, 19 (1985).

${ }^{30}$ G. Travaglini, P. Wachter, J. Marcus, and C. Schlenker, Solid State Commun. 37, 599 (1981).

${ }^{31}$ P. Monceau, L. Bernard, R. Currat, F. Levy, and J. Rouxel, Physica B 136, 352 (1986).

32 J. P. Pouget, B. Hennion, C. Escribe-Filippini, and M. Sato, Phys. Rev. B 43, 8421 (1991).

${ }^{33}$ D. Berner, H. P. Geserich, W. Brütting, and W. Rieß (unpublished).

${ }^{34}$ P. Gressier, A. Meerschaut, J. Rouxel, and M.-H. Whangbo, in Charge Density Waves in Solids, edited by G. Hutiray and J. Sólyom, Lecture Notes in Physics Vol. 217 (Springer, Berlin, 1985), p. 43.

${ }^{35}$ S. Thieme, L. Degiorgi, G. Grüner, and P. Wachter, Proceedings of the International Conference on Synthetic Metals, Seoul, 1994 [Synth. Met. (to be published)].

${ }^{36} \mathrm{G}$. Travaglini and P. Wachter, in Charge Density Waves in Solids (Ref. 34), p. 115. 